\title{
Les dynamiques d'innovation dans les industries aérospatiales et de défense : nouveaux modèles, nouvelles pratiques, nouveaux usages
}

\section{Innovation dynamics in aerospace and defense industries: new models,} new practices, new uses

\author{
Pierre Barbaroux ${ }^{1,2,3}$, Zeting Liu ${ }^{4}$ \\ ${ }^{1}$ Centre de recherche de l'armée de l'air, Ecole de l'air, Salon de Provence, France, pierre.barbaroux@ecole-air.fr \\ ${ }^{2}$ Chaire « Cyber Résilience Aérospatiale » de l'Armée de l'air. \\ ${ }^{3}$ Université de Strasbourg, BETA, CNRS UMR 7522. \\ ${ }^{4}$ Université du Littoral Cote d'Opale, CLERSE, CNRS UMR 8019, France, zeting.liu@hotmail.fr
}

RÉSUMÉ. Les industries aérospatiales et de défense (A\&D) connaissent des transformations profondes. Les technologies changent, les compétences techniques et les comportements d'usage également, les pratiques évoluent. Cet article éditorial présente les enjeux et les défis majeurs accompagnant la dynamique d'innovation à l'œuvre dans les industries A\&D en distinguant trois niveaux d'analyse : le secteur industriel, la firme et les pratiques individuelles et collectives.

ABSTRACT. Aerospace and defense (A\&D) industries are changing. Technologies are deeply transforming firms' technical competences, user-behaviours and managerial practices. This editorial article presents the major issues and challenges supported by the innovation dynamics operating within A\&D industries by distinguishing three levels of analysis: the industrial sector, the firm and individual and collective practices.

MOTS-CLÉS. Innovation, Industries aérospatiales, gestion des connaissances, brevets, apprentissage, compétences, pratiques d'usages.

KEYWORDS. Innovation, aerospace industries, knowledge management, patents, learning, competences, user practices.

\section{L’innovation comme moteur du changement dans les industries aérospatiales et de défense}

Longtemps pionniers en matière d'innovation technologique, de design organisationnel et de développement des compétences techniques et des pratiques d'usage [HUG 04], les acteurs des industries aérospatiales et de défense (A\&D) sont aujourd'hui très sensibles aux changements externes affectant leurs modèles d'affaires et leurs capacités d'innovation. Les révolutions numérique et écologique, l'intensification de la concurrence internationale, la variété et la dualité des sources de connaissances affectent en effet durablement leurs processus de conception, d'usage et de création de valeur. L'ensemble des composantes de l'architecture des systèmes aérospatiaux et de défense est concerné : les vecteurs (e.g., matériaux composites, aéronefs autonomes ou pilotés à distance, constellations de satellites), les capteurs (e.g., technologies de détection et de navigation, furtivité, sense making), les systèmes de commandement et de communication (e.g., numérisation et cybersécurité des espaces de travail), les compétences individuelles et collectives (e.g., conception et développement, normes et valeurs, usages) ainsi que le cadre réglementaire et juridique (e.g., nouveaux services aériens et spatiaux, nouvelles formes de mobilité) changent, remodelant durablement le paysage technologique et commercial dans lequel évoluent les acteurs des industries A\&D.

Pour de nombreux commentateurs et analystes, ces transformations sont potentiellement disruptives pour les modèles d'affaires et les compétences techniques existants [MAI 17]. L'intégration fonctionnelle des technologies de production, de communication et d'aide à la décision numériques, automatisées et intelligentes ouvrent des opportunités pour les entreprises et les utilisateurs installés, tout en menaçant leurs positions concurrentielles, leurs modèles de Recherche \& Développement 
(R\&D), voire leur culture industrielle [SER 18]. L'arrivée de nouveaux acteurs et la diffusion de pratiques et de comportements nouveaux, en particulier issus de l'économie numérique, justifient un effort d'adaptation et de conduite du changement de la part des acteurs installés (i.e., Etats, entreprises, communautés scientifiques et utilisateurs). Dans ce contexte, l'innovation est une stratégie efficace leur permettant de renouveler leurs modèles d'affaires, leurs compétences techniques, leurs organisations et leurs pratiques d'usages [BAR 17].

\section{Les trois niveaux d'analyse de l'innovation: inter-organisationnel, organisationnel, individuel}

Désignant l'invention et la commercialisation de nouveaux produits, services, technologies, organisations et processus, l'innovation relève de l'articulation de trois processus de connaissances : la génération, l'application et la valorisation des connaissances [BAR 16]. La capacité des parties prenantes du processus d'innovation de créer de nouvelles connaissances, de les incorporer dans des biens et des services susceptibles de créer de la valeur pour des clients ou des utilisateurs, et de définir un régime d'appropriation viable en vue de percevoir et de distribuer les revenus issus de leur commercialisation, est en effet essentielle. Or, la génération, l'application et la valorisation des connaissances opèrent à différentes échelles : inter-organisationnelle (au niveau du secteur ou d'un écosystème plus large), organisationnelle (au niveau de la firme et des organisations publiques) et individuelle (au niveau des équipes et des individus).

La compréhension des dynamiques d'innovation suppose donc de distinguer les échelles d'analyse et, éventuellement, de les articuler.

A l'échelle inter-organisationnelle, les firmes nourrissent une dynamique collective qui se traduit par l'accumulation de connaissances nouvelles, le développement de compétences techniques plus performantes, la mise en oeuvre de régimes d'appropriation adaptés et l'extension de la taille des marchés. La mesure des processus d'accumulation et de valorisation des connaissances est ici primordiale et le recours aux données de brevets ou aux volumes de dépenses en R\&D est une stratégie courante en matière de collecte du matériau empirique à partir duquel construire un indicateur de mesure de l'intensité en connaissances d'un secteur ou d'une industrie [SER 08]. A l'échelle des firmes, l'innovation procède du développement de capacités spécifiques (i.e., absorption, design, collaboration etc.) et du déploiement de modèles de gestion des processus d'innovation adaptés aux besoins et aux structures des marchés sur lesquels elles opèrent (e.g., innovation ouverte, innovation centrées sur les utilisateurs, innovation participative etc.). L'ensemble des organisations publiques et privées est concerné par le renouvellement des modèles de gestion de l'innovation, de management de projets ou d'acquisition de capacités innovantes. L'objectif recherché est bien souvent un raccourcissement des phases amonts du cycle de vie des innovations (e.g., étude de faisabilité, expérimentations, démonstrateurs technologiques et preuves de concept) et une plus grande agilité relationnelles et commerciales [NOB 04]. A l'échelle des individus et des équipes, l'innovation repose sur des processus d'apprentissage conduisant les concepteurs, les ingénieurs, les développeurs et les utilisateurs à enrichir leur portefeuille de compétences mobilisées lors des différentes phases du cycle de vie des innovations (e.g., idéation, conception, développement, exploitation, usages, maintenance etc.). Il s'agit alors de comprendre comment l'intégration des compétences d'usage, de conception et de développement, d'une part, génére des connaissances expérimentales utiles relatives aux besoins et aux capacités futures des utilisateurs et des concepteurs et, d'autre part, nourrit le processus d'idéation à travers l'élimination des "mauvaises idées 》 et la génération de preuves de concept viables susceptibles de justifier un effort supplémentaire (e.g., maturation technologique et industrialisation).

Ce découpage multi-échelle offre une grille de lecture des dynamiques d'innovation à l'oeuvre au sein des industries aérospatiales et de défense. C'est cette grille que nous utilisons pour organiser la structure du présent numéro thématique. 


\section{Présentation du contenu du numéro thématique}

Les trois premiers articles concernent l'étude des dynamiques d'innovation à l'échelle interorganisationnelle (i.e., industrielle ou sectorielle). Ces trois articles analysent les processus de création et de diffusion des connaissances des entreprises A\&D à travers différentes interpretations des activités de $R \& D$ et des brevets.

Cécile Fauconnet propose ainsi d'étudier l'évolution des industries A\&D de 1945 à 2015 en insistant sur la contribution des connaissances scientifiques à la dynamique des innovations technologiques sur la longue période. A partir d'un travail bibliométrique portant sur des données de brevets, l'auteure montre comment la littérature non brevetable (publication scientifique) est de plus en plus citée dans les brevets, traduisant une modification profonde de la structure des innovations au sein des industries A\&D sur le modèle de l'économie de la connaissance.

François-Xavier Meunier approfondit le travail précédent en introduisant une méthode (i.e., les Systèmes de Connaissances Technologiques ; SCT) permettant de repérer empiriquement les systèmes de connaissances qui structurent le «paysage technologique» au sein des industries de défense. Appliquée à l'analyse des flux de connaissances matérialisés à partir des citations de brevets, cette méthode met en évidence les relations de dépendance, d'interdépendance et de dominance dans le processus de production de la connaissance des entreprises A\&D.

Cécile Fauconnet, Didier Lebert, Célia Zyla et Sylvain Moura analysent plus particulièrement la relation entre dualité technologique et dualité productive d'une part, et les mesures de la cohérence technologique et du ratio d'exploitation d'autre part. En établissant une typologie des entreprises de la Base Industrielle et technologique de Défense (BITD) à partir de leur relations (relateness) technologiques, les auteurs montrent que l'hypothèse d'une relation positive entre la dualité technologique et la dualité productive dans ce secteur n'est pas valide. A contrario, l'analyse des stratégies de R\&D approchées par la relation entre la diversité technologique et le ratio d'exploration est plus prometteur.

Les deux articles suivants explorent les comportements stratégiques des firmes innovantes à l'échelle organisationnelle.

Florin Paun étudie les nouvelles pratiques mises en œuvre par les entreprises A\&D. Il montre comment les nouveaux modèles collaboratifs de gestion de l'innovation, ouverts et agiles, modifient l'architecture des relations entre acteurs et génèrent un raccourcissement significatif des cycles d'innovation. La multiplication des illustrations et des témoignages collectés par l'auteur témoigne d'un bouleversement fondamental de la structure relationnelle ainsi que des stratégies d'exploration des connaissances au sein des industries A\&D. Ce faisant, l'auteur parvient à articuler les échelles d'analyse, partant des stratégies d'innovation des firmes pour explorer leurs implications sur les cycles de l'innovation à court, moyen et long termes.

Guillaume Blum et Mehran Ebrahimi enrichissent la perspective précédente en portant l'effort de recherche sur une tendance lourde en matière de renouvellement des modèles de gestion de l'innovation dans les organisations A\&D : l'écologie. A partir d'une enquète menée auprès d'une trentaine d'acteurs de la grappe aéronautique Québécoise, les auteurs décrivent les processus de connaissances sous-tendant l'éco-innovation que représente 1' « avion vert ». Ils montrent alors que si les connaissances écologiques sont perçues par les acteurs (e.g., ingénieurs, chefs de projets, entrepreneurs) comme étant neutres au regard des critères de performance appliqués dans le champ de l'ingénieirie des systèmes complexes, elles suscitent toutefois l'apparition de nouvelles contraintes. L'article suggère alors que les préoccupations environnementales vont durablement modifier les comportements des acteurs à travers la transformation des achitectures de connaissances sur lesquelles reposent leurs modèles d'affaires. 
Les trois derniers articles s'intéressent à la dynamique d'innovation opérant à l'échelle des équipes et des individus qui les composent (utilisateurs et concepteurs).

Cyril Camachon et Pierre Barbaroux interrogent ainsi l'impact de la numérisation des aéronefs de dernière génération sur la nature et la variété des compétences des opérateurs, ainsi que sur les processus d'acquisition de ces compétences. A partir d'un matériau empirique récolté au cours de plusieurs études et contrats de recherche instiutionnels (armée de l'air française), les auteurs montrent comment la numérisation des espaces de travail (i.e., glass cockpits numérisés) modifie la nature des compétences techniques fondamentales des pilotes («basiques du vol») et justifie un travail de réingénieirie des processus de formation des pilotes. Les auteurs discutent ensuite des conditions favorables pour que l'introduction des techniues de simulation embarquée (embedded simulation), relevant de la combinaison des techniques de simulation réelle et virtuelle, apporte des ressources supplémentaires permettant d'optimiser la formation des futurs pilotes.

Prolongeant l'analyse précédente des changements induits par les innovations technologiques sur les utilisateurs des systèmes aéronautiques, Mick Salomone, Ludovic Fabre et Pierre Barbaroux explorent les effets de la révolution numérique (e.g., numérisation automatisation) sur les comportements et la performance des opérateurs. A partir d'une caractérisation des processus cognitifs des individus (ingénierie cognitive), les auteurs suggèrent que l'un des effets induits par la numérisation des espaces de travail est une augmentation de la fatigue cognitive des opérateurs. Dans ce cadre, les auteurs montrent comment l'étude scientifique des mécanismes cognitifs accompagnant l'apparition de la fatigue cognitive peut permettre, d'une part, d'améliorer notre compréhension des stratégies de mitigation mises en place par les opérateurs pour maintenir un haut niveau de performance. D'autre part, les auteurs suggèrent que la connaissance scientifique ainsi produite rend attractive la conception d'interfaces intelligentes, capables de s'adapter à l'état cognitif de l'opérateur, grâce au monitoring « temps-réel» de son comportement et à la détection des signes de survenance de la fatigue cognitive.

Le troisième article insiste sur le rôle de la recherche en matière de génération de connaissances lors des phases amont du processus d'innovation (conception), mais en adoptant le point de vue des sciences pour l'ingénieur. Bertrand Kirsch et Olivier Montagnier étudient ainsi les contraintes physiques associées au développement d'un aéronef piloté à distance de grande envergure, doté d'une voilure fixe et d'une capacité d'endurance élevée (drone HALE solaire). Les auteurs explicitent les difficultés scientifiques et techniques posées par le développement d'un aéronef de ce type en soulignant l'existence d'un verrou structurel et aérodynamique (i.e., Masse vs. Endurance de l'aéronef) suceptible d'être lever grâce à une technique appelée «tissage aéroélastique ». Les auteurs montrent alors comment la recherche appliquée peut nourrir la dynamique d'innovation à travers la production de connaissances expérimentales indispensables au développement d'une innovation technologique dont les applications apparaissent prometteuses et variées.

Le numéro thématique s'achève par un "article-témoignage » portant sur rôle de l'innovation au cours des phases d'exploitation et de maintien en condition opérationnelle (MCO) du cyle de vie des systèmes militaires. Nicolas Hué, Walter Arnaud et Christophe Grandemange rendent ainsi compte de leurs expériences sur la façon dont les innovations ont été intégrées dans la stratégie de la Délégation Générale de l'Armement (DGA) au fil des étapes successives d'un Programme d'Armement. Ils exposent ainsi le point de vue de praticiens éclairés sur les enjeux et les perspectives relatifs à la diffusion des innovations au sein de la BITD.

$* * *$

Les neuf articles qui composent ce numéro thématique de la revue Technologie \& Innovation illustrent la façon dont la dynamique d'innovation opère à l'échelle des industries, des firmes et des individus utilisateurs et concepteurs de systèmes aéronautiques et de défense. L'innovation, enracinée dans des processus de connaissances, est à la fois le moteur du changement industriel et une stratégie 
permettant aux acteurs individuels et organisationnels, de s'adapter. Pour en comprendre les déterminants et les effets, ce numéro a privilégié la variété et la pluridiciplinarité des points de vue et des analyses. Appliquée à l'étude d'un objet aussi complexe que l'innovation dans les industries aérospatiales et de défense, cette diversité thématique et méthodologique enrichit notre compréhension des pratiques des acteurs, ainsi que de leurs implications à différentes échelles. En encourageant le dialogue entre les disciplines et les communautés (e.g., chercheurs, ingénieurs, concpeteurs, utilisateurs), ce numéro participe du décloisonnement des paradigmes de l'ingénierie des systèmes, de la science et des usages qui gouvernent aujourd'hui les relations entre Technologie et Innovation.

Bonne lecture !

\section{Bibliographie}

[BAR 16] BARBAROUX, P., ATTOUR, A., SCHENK, E, Gestion des Connaissances et Innovation : Interaction, Collaboration, Ouverture, ISTE Publishing, 2016.

[BAR 17] BARBAROUX, P. Apprendre et Innover : Une exploration des modalités d'adaptation et de conduite du changement économique et organisationnel, Mémoire pour l'Habilitation à Diriger des Recherches, Pole Européen de gestion et d'Economie (PEGE), Université de Strasbourg, Juin 2017, 223 pages.

[HUG 04] HUGUES, T.P. American Genesis: A Century of Invention and Technological Enthusiasm, 1870-1970, The University of Chicago Press, Chicago \& London, 2004 ( $2^{\text {nd }}$ edition).

[MAI 17] MAIRE, S. «Aéronautique : l'avenir du secteur passera par l'innovation et la transformation digitale », Les Echos, 13/12/2017 (consulté le 05/03/2018 ; https://www.lesechos.fr/idees-debats/cercle/cercle-177101-aeronautiquelavenir-du-secteur-passera-par-linnovation-et-la-transformation-digitale-2138084.php).

[NOB 04] NOBELIUS D., "Towards the sixth generation of R\&D management », International Journal of Project Management, 2004, Vol. 22, p. 336-375.

[SER 08] SERFATI, C., "Le rôle de l'innovation de défense dans le système national d'innovation de la France ", Innovations, revue d'Economie et de Management de l'Innovation, 2008, vol. 28, n² , p. 61-83.

[SER 18] SERRIS J., «Introduction », Enjeux Numériques, Une série des annales de l’Ecole des Mines, $2018, \mathrm{~N}^{\circ} 1\left(\mathrm{n}^{\circ}\right.$ thématique : «L’intelligence artificielle : un enjeu d'économie et de civilisation ?», p. 6-9. 